\title{
EDITORIAL
}

\section{PROMOCIÓN DE LA SALUD BASADA EN LA EVIDENCIA}

\author{
Joan R Villalbí \\ Institut Municipal de Salut Pública, Ajuntament de Barcelona
}

Hay tres componentes principales de las iniciativas de educación sanitaria y promoción de la salud basadas en la evidencia: su fundamento en un modelo teórico sólido, el tener un contenido definido y repetible, y el lograr una eficacia conocida y relevante. Sin ellas, la educación sanitaria y la promoción de la salud se convierten fácilmente en retórica vacía, cuando no en coartada para la pasividad.

Un modelo teórico sólido permite sustentar propuestas plausibles. Del mismo modo que las ciencias básicas proporcionan el sustento para las intervenciones farmacológicas, las intervenciones dirigidas a modificar el comportamiento precisan de una base conceptual sólida. Las aportaciones de Bandura $^{1}$ se han revelado como las más apropiadas para el trabajo en promoción de la salud y educación sanitaria, al proporcionar una perspectiva global del comportamiento humano, integrando las aportaciones de diversas disciplinas.

Tener un contenido definido y repetible resulta esencial para que un programa exista realmente. Si los contenidos son genéricos, no están protocolizados, o dependen de la creatividad de quien los desarrolla, es probable que el programa no se aplique de forma sistemática ${ }^{2}$. Muchas acciones de salud

Correspondencia:

Joan R Villalbí

Institut Municipal de Salut Pública

Ajuntament de Barcelona

Pl Lesseps 1

08023 Barcelona

Correo electrónico: jrvillal@imsb.bcn.es pública se basan en rutinas fáciles de reproducir, que así pueden ser aplicadas por personal poco cualificado, o bien por personal cualificado que las incorpora a otro cometido principal. Incorporar acciones de educación para la salud y promoción de la salud a la dinámica de los servicios de salud pública exige un esfuerzo previo de estandarización. Además, se necesita un contenido bien definido si se ha de monitorizar luego su cumplimiento efectivo.

Una eficacia conocida y relevante es la clave de una intervención efectiva. Demostrar la utilidad de las acciones es un requisito básico para que puedan ampliarse y extenderse. Además, en el ambiente de feroz competencia por unos recursos limitados que caracteriza a las administraciones sanitarias desde hace una década, los programas que no puedan invocar una eficacia clara y acreditada no sobreviven. Por otra parte, dedicar algún tiempo a escarbar en las bases de datos bibliográficas permite identificar éxitos y fracasos ajenos en los cuales inspirarse, con un esfuerzo relativamente pequeño. Todos los que trabajamos en este campo le debemos mucho a Larry Green y a sus libros ${ }^{3}$.

En este número de la Revista Española de Salud Pública se presentan dos trabajos que muestran la vitalidad de la promoción de la salud en nuestro país. Por un lado, la detallada revisión de los trabajos de promoción de la salud en jóvenes desarrollada por Mariano Hernán y sus colaboradores en la Escuela Andaluza de Salud Pública ${ }^{4}$ : en ella se ilustran algunas de las ideas enunciadas antes. Por otra parte, el estudio de Gabriel J Díaz 
Grávalos, sobre los factores relacionados con la educación sanitaria en la consulta médica en los centros de atención primaria de Galicia, muestra las dificultades para integrar elementos de educación para la salud en el trabajo cotidiano de un profesional que siente que su misión principal es otra, y que apenas dispone de tiempo para cubrirla ${ }^{5}$. Algo que ha merecido recientemente un interesante debate ético en nuestro medio ${ }^{6}$. A ellos se añade el artículo de Valentín Gavidia que traspone al español los conceptos de la escuela promotora de salud, desarrollados años atrás por diversos autores anglosajones ${ }^{7}$, y que han mostrado su utilidad para mantener una visión más integral de los esfuerzos de prevención en la escuela.

Ante esta muestra de vitalidad, parece oportuno recordar otro aspecto crucial: la necesidad de ampliar la cobertura de aquellas iniciativas de valor demostrado. Es bien cierto que hay programas de educación sanitaria que, tras ser probados de forma piloto, se extienden a gran escala alcanzando coberturas amplias. Pero desgraciadamente aún es frecuente que muchos excelentes proyectos queden como iniciativas aisladas, o circunscritos a un ámbito demasiado local. Es evidente que su difusión en los medios profesionales ayuda: la revista Idea Prevención en el campo de las adicciones, o los registros de proyectos y materiales inventariados por la Escuela Andaluza de Salud Pública han contribuido a la extensión de la excelencia en promoción de la salud. Pero sigue habiendo dificultades para ampliar la cobertura de los programas con éxito, probablemente por la falta de un esquema de difusión y financiación apropiado. Quizás la estructura autonómica del país y la existencia de fondos para proyectos innovadores favorece que surjan iniciativas, pero la falta de recursos para la extensión a mayor escala de los proyectos con éxito y las fronteras administrativas no favorecen su extensión. Habrá que ver como facilitarlo.

Finalmente, se comprueba cómo muchas iniciativas se centran en la intervención sobre un factor de riesgo tomado de manera aislada, abordado de forma persuasiva, y limitado al comportamiento. Sabemos que muchos factores de riesgo son sensibles a abordajes más globales, pero éstos precisan de una intervención más política, para la que se requiere la voluntad de afrontar grupos de interés poderosos. En España necesitamos un mayor énfasis en el desarrollo de políticas públicas saludables. El ejemplo de otros países sugiere que debemos prestar una mayor atención al proceso de construcción de políticas, y no desdeñar el papel de lobby que pueden jugar determinadas organizaciones para conseguir progresos en el desarrollo de políticas públicas que favorezcan la salud $^{8}$. La experiencia lograda en el terreno de la prevención del tabaquismo es ilustrati$\mathrm{va}^{9}$, y debe servir para generar una reflexión, capaz de conducir a la acción.

\section{BIBLIOGRAFÍA}

1. Bandura A. Pensamiento y acción. Fundamentos sociales. Barcelona: Martínez Roca Editor; 1987.

2. Hawe P, Degeling D, Hall J. Evaluating health promotion. Artarmon: MacLennan and Petty Pty Limited; 1990.

3. Green LW, Kreuter MW, Deeds SG, Partridge KB. Health education planning: a diagnostic approach. Palo Alto: Mayfield Publishing; 1980.

4. Hernán M, Ramos M, Fernández A. Revisión de los trabajos publicados sobre promoción de la salud dirigidos a los jóvenes españoles. Rev Esp Salud Pública 2001; 75: Poner números de páginas.

5. Díaz-Grávalos GJ. Factores relacionados con la práctica de educación sanitaria por los médicos de atención primaria de Galicia, España. Rev Esp Salud Pública 2001; 75: Poner números de páginas.

6. Altisent R, Brotons C, González R, Serrat D, Júdez J, Gracia D. Etica de la actividad preventiva en atención primaria. Med Clin (Barc) 2001; 117: $740-50$.

7. Gavidia V. La transversalidad y la escuela promotora de salud. Rev Esp Salud Pública 2001; 75: Poner números de páginas.

8. Subirats J. El análisis de las políticas públicas. Gac Sanit 2001; 15: 259-264.

9. Villalbí JR, López V. La prevención del tabaquismo como problema político. Gac Sanit 2001; 15 : 265-272.

Rev Esp Salud Pública 2001, Vol. 75, N. ${ }^{\circ} 6$ 